\title{
Cancer therapy and renal injury
}

\author{
Robert W. Schrier \\ Department of Medicine, University of Colorado Health Sciences Center, Denver, Colorado, USA \\ J. Clin. Invest. 110:743-745 (2002). doi:10.1172/JCI200216568.
}

Cisplatin and other platinum derivatives are among the most effective chemotherapeutic agents against solid tumors including ovarian, head and neck carcinomas, and germ cell tumors. The major side effect of cisplatin is nephrotoxicity. While several antineoplastic agents frequently exhibit nephrotoxicity, the platinum derivatives are among the most frequent compounds leading to renal injury (1). There also can be synergistic nephrotoxic effects of cisplatin with other chemotherapeutic agents such as the taxol compounds (2). Moreover, in hematopoietic cell transplantation (HCT), there appears to be a dramatic interaction between chemotherapeutic agents, such as cisplatin, and immunosuppressive agents such as cyclosporine. Specifically, nephrotoxicity, as assessed by a $25 \%$ loss of glomerular filtration rate (GFR), has been observed in $56 \%$ of autologous HCTs, in which immunosuppressive therapy is not used, but in more than $90 \%$ of allogeneic HCT, which requires immunosuppression $(3,4)$. Moreover, mortality is $7 \%$ in autologous HCTs in which cisplatin and

Address correspondence to: Robert W. Schrier, Department of Medicine, University of Colorado Health Sciences Center, Box B178, 4200 East 9th Avenue, Denver, Colorado 80262, USA.

Phone: (303) 315-7765; Fax: (303) 315-7702;

E-mail: Robert.Schrier@uchsc.edu.

Conflict of interest: No conflict of interest has been declared.

Nonstandard abbreviations used: hematopoietic cell transplantation (HCT); glomerular filtration rate (GFR); acute renal failure (ARF); superoxide dismutase (SOD); reduced glutathione (GSH); glutathione peroxidase $(\mathrm{GP})$; superoxide $\left(\mathrm{O}_{2}^{-}\right)$; renal blood flow (RBF); reactive oxygen intermediates (ROI). other antineoplastic drugs are used without immunosuppression. In contrast, the mortality rises to $58 \%$ in allogeneic HCTs when the antineoplastic drugs are combined with immunosuppressive drugs, particularly calcineurin inhibitors, such as cyclosporine.

Thus, there is a pressing need for ways to protect the kidney while administering effective chemotherapeutic agents, such as cisplatin. Ramesh and Reeves in this issue of the JCI provide evidence that TNF- $\alpha$ is a critical factor in mediating chemokine and cytokine expression and renal injury in a murine model of cisplatin nephrotoxicity (5). Using inhibitors of TNF- $\alpha$ release and activity as well as knockout mice, they show here that cisplatin nephrotoxicity can be attenuated but not totally abolished.

\section{TNF- $\alpha$ in renal inflammation and injury}

Figure 1 shows a schema of events by which TNF- $\alpha$ can contribute to renal injury and acute renal failure (ARF) after cisplatin administration. Thus, cisplatin may initially reduce the levels and availability of endogenous oxygen

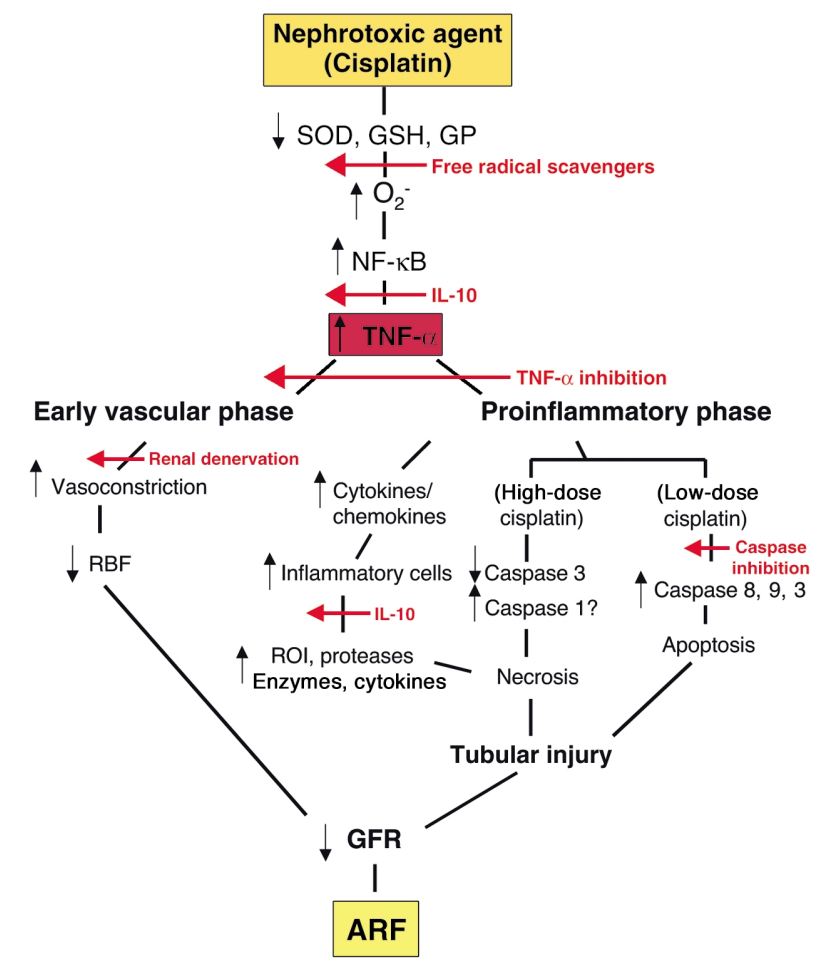

Figure 1

Events proposed to cause nephrotoxic ARF. As indicated in the text, TNF- $\alpha$ participates in both an early, vascular phase and a later, proinflammatory phase in this response. Red arrows indicate points in the pathway where various protective therapies appear to interfere with nephrotoxicity. 
radical scavengers such as superoxide dismutase (SOD), much as administration of LPS can do $(6,7)$. In addition, reduced glutathione (GSH) and glutathione peroxidase (GP) activities in cisplatin-treated tissues may contribute to increased generation of superoxide $\left(\mathrm{O}_{2}^{-}\right)$and other oxygen radicals $(8,9)$, and, indeed, cisplatin has been demonstrated to generate $\mathrm{O}_{2}{ }^{-}$ anion in a cell-free system (10). The resultant oxidant stress activates the transcription factor NF- $\kappa \mathrm{B}$, which in turn stimulates the production of TNF- $\alpha(11,12)$.

TNF- $\alpha$ induction occurs early in ischemia/reperfusion injury, indicating that TNF- $\alpha$ is produced by intrinsic kidney cells, rather than by infiltrating leukocytes, which only accumulate in the kidney later, during the proinflammatory phase of renal injury (12). During endotoxemia as well, the increased production of TNF- $\alpha$ results in an early vascular phase characterized by renal vasoconstriction, reduced renal blood flow (RBF) and GFR, in association with the activation of the sympathetic and renin-angiotensin systems (13, 14). The role of the increased sympathetic activity in this early vascular phase is supported by the protective effect of renal denervation (14). Specifically, in a mouse model of endotoxemic ARF, TNF- $\alpha$ neutralization affords renal protection in the absence of hypotension, renal parenchymal leukocyte infiltration, glomerular fibrin, apoptosis, or morphological changes (13). These results support the view that the early effect of TNF- $\alpha$ is primarily mediated by renal vasoconstriction.

From the new work by Ramesh and Reeves (5), it is clear that cisplatinrelated nephrotoxicity can also lead to a later, proinflammatory phase of TNF- $\alpha$-mediated renal injury. This phase is characterized by accelerated production of cytokines and chemokines by all types of renal cells (15). Increased TNF- $\alpha$ leads to the recruitment and accumulation of inflammatory cells including neutrophils, monocytes, natural killer cells and $\mathrm{T}$ lymphocytes as shown by Ramesh and Reeves and others $(16,17)$. These white blood cells secrete lysosomal enzymes, reactive oxygen intermediates (ROI) and proteases, which can directly injure surrounding renal tissue. In addition, the activation of these leukocytes may lead to further production of cytokines or chemokines and greater recruitment of leukocytes to the focus of inflammation, thus amplifying the renal inflammatory response (16). It would be interesting to examine whether this proinflammatory-related renal injury with cisplatin can be induced in a leukocytedepleted murine model.

Finally, depending on the dose of cisplatin, renal tubular cells succumb to either necrotic or apoptotic cell death $(8,18)$. At lower doses, the proapoptotic initiator caspases 8 and 9 , and the executioner caspase 3 are activated and result in apoptosis as indicated by cell shrinkage, chromatin condensation, nuclear fragmentation, and DNA laddering on gel electrophoresis $(18,19)$. In contrast, higher doses of cisplatin are accompanied by a decline in caspase 3 activity and widespread cell necrosis, characterized by cytosolic swelling, loss of plasma membrane integrity, and a smear pattern on DNA gel electrophoresis $(8,18)$. The proinflammatory caspase 1 has been implicated in ischemic necrotic cell death (20). This enzyme converts prointerleukin-1 $\beta$ (IL-1 $\beta$ ) and prointerleukin-18 (IL-18) to their respective active forms. Interestingly, Ramesh and Reeves show here that IL- $1 \beta$ is induced by cisplatin, in parallel with an increase in tubular necrosis (5). In addition, IL-18 has been shown to be a mediator of necrosis in ischemic ARF (20). Caspase 1 activation by cisplatin does not appear to occur in cultured renal epithelial cells $(8,19)$, but this possibility has not been examined in vivo. Therefore, caspase 1 could potentially contribute to the inflammatory process and necrotic cell death observed in cisplatin nephrotoxicity. Although the relative roles of apoptosis versus necrosis in cisplatin nephrotoxicity remain to be defined, the study of caspase inhibition in the later phase of cisplatin nephrotoxicity would be of interest.

\section{Clinical management of nephrotoxicity}

As shown in Figure 1, various agents and maneuvers used to block certain steps of this proposed pathway have been demonstrated to be beneficial when administered at the time of cisplatin administration or shortly thereafter. These treatments include the addition of free radical scavengers, such as dimethylthiourea or lecithinized SOD (21), and IL-10, which can inhibit neutrophil and macrophage function both directly and by blocking the transcription of TNF- $\alpha$ (12). The present report confirms the protective effect of TNF- $\alpha$ antagonists or knockouts, consistent with work in a model of endotoxemic ARF, where either renal denervation or treatment with a soluble form of the TNF- $\alpha$ receptor has been shown to prevent the early reduction in GFR $(13,14)$. Finally, caspase inhibition has been shown to provide marked protection against apoptotic cell death in vitro (19).

Because the occurrence of ARF associated with sepsis, ischemia, or both is not predictable, early proactive intervention is generally not feasible. Still, even when treatment cannot be applied before such an insult, early protective intervention helps attenuate renal injury. Thus, the substantial incidence of nephrotoxicity which has been observed in patients treated with cisplatin may be markedly reduced by the concomitant administration of therapeutic agents that inhibit the release or activity of TNF- $\alpha$.

1. Safirstein, R.L. 2001. Renal diseases induced by anti-neoplastic agents. In Diseases of the kidney and urinary tract. R.W. Schrier, editor. Lippincott Williams and Wilkins. Philadelphia, Pennsylvania, USA. 1175-1188.

2. Merouani, A., Davidson, S.A., and Schrier, R.W 1997. Increased nephrotoxicity of combination taxol and cisplatin chemotherapy in gynecologic cancers as compared to cisplatin alone. Am. J. Nephrol. 17:53-58.

3. Merouani, A., Shpall, E.J., Jones, R.B., Archer, P.G., and Schrier, R.W. 1996. Renal function in high dose chemotherapy and autologous hematopoietic cell support treatment for breast cancer. Kidney Int. 50:1026-1031.

4. Parikh, C.R., et al. 2002. Renal dysfunction in allogeneic hematopoietic cell transplantation. Kidney Int. 62:566-573.

5. Ramesh, G., and Reeves, W.B. 2002. TNF- $\alpha$ mediates chemokine and cytokine expression and renal injury in cisplatin nephrotoxicity. J. Clin. Invest. 110:835-842. doi:10.1172/ JCI200215606.

6. Davis, C.A., Nick, H.S., and Agarwal, A. 2001. Manganese superoxide dismutase attenuates cisplatin-induced renal injury: importance of superoxide. J. Am. Soc. Nephrol. 12:2683-2690.

7. Wang, W., et al. 2002. Decreased expression of extracellular superoxide dismutase (EC-SOD) in the kidney during endotoxemic acute renal failure (ARF) in mice (abstract). J. Am. Soc Nephrol. In press. 
8. Lau, A.H. 1999. Apoptosis induced by cisplatin nephrotoxic injury. Kidney Int. 56:1295-1298.

9. Sugiyama, S., et al. 1989. Adverse effects of anti-tumor drug, cisplatin, on rat kidney mitochondria: disturbances in glutathione peroxidase activity. Biochem. Biophys. Res. Commun. 159:1121-1127.

10. Masuda, H., Tanaka, T., and Takahama, U. 1994. Cisplatin generates superoxide anion by interaction with DNA in a cell- free system. Biochem. Biophys. Res. Commun. 203:1175-1180.

11. Barnes, P.J., and Karin, M. 1997. Nuclear factorkappaB: a pivotal transcription factor in chronic inflammatory diseases. N. Engl. J. Med. 336:1066-1071.

12. Deng, J., et al. 2001. Interleukin-10 inhibits ischemic and cisplatin-induced acute renal injury. Kidney Int. 60:2118-2128.
13. Knotek, M., et al. 2001. Endotoxemic renal failure in mice: role of tumor necrosis factor independent of inducible nitric oxide synthase. Kidney Int. 59:2243-2249.

14. Wang, W., et al. 2002. Protective effect of renal denervation on normotensive endotoxemiainduced acute renal failure (ARF) in mice. $A m$. J. Physiol. Renal. Physiol. 283:583-587.

15. Segerer, S., Nelson, P.J., and Schlondorff, D. 2000. Chemokines, chemokine receptors, and renal disease: from basic science to pathophysiologic and therapeutic studies. J. Am. Soc. Nephrol. 11:152-176.

16. Rovin, B.H., and Phan, L.T. 1998. Chemotactic factors and renal inflammation. Am. J. Kidney Dis. 31:1065-1084.

17. Kelly, K.J., Meehan, S.M., Colvin, R.B. Williams, W.W., and Bonventre, J.V. 1999. Protection from toxicant-mediated renal injury in the rat with anti- CD54 antibody. Kidney Int 56:922-931.

18. Lieberthal, W., Triaca, V., and Levine, J. 1996. Mechanisms of death induced by cisplatin in proximal tubular epithelial cells: apoptosis vs. necrosis. Am. J. Physiol. 270:F700-F708.

19. Kaushal, G.P., Kaushal, V., Hong, X., and Shah, S.V. 2001. Role and regulation of activation of caspases in cisplatin-induced injury to renal tubular epithelial cells. Kidney Int 60:1726-1736.

20. Melnikov, V.Y., et al. 2001. Impaired IL-18 processing protects caspase-1-deficient mice from ischemic acute renal failure. J. Clin. Invest. 107:1145-1152.

21. Matsushima, H., Yonemura, K., Ohishi, K., and Hishida, A. 1998. The role of oxygen free radicals in cisplatin-induced acute renal failure in rats. J. Lab. Clin. Med. 131:518-526. 